\title{
Is adrenal suppression in asthmatic children reversible? A case series
}

\author{
E W Zöllner, PhD, MMed, MB ChB, DCH, DTM\&H, DCH \\ Endocrine Unit, Department of Paediatrics, Tygerberg Children's Hospital and Faculty of Medicine and Health Sciences, Stellenbosch University, \\ Cape Town, South Africa
}

Corresponding author: E W Zöllner (zollner@sun.ac.za)

Background. Six hypocortisolaemic asthmatic children on steroids given at physiological doses were identified during a previous study. Objectives. To establish whether hypothalamic-pituitary-adrenal axis suppression (HPAS) could be reversed in hypocortisolaemic asthmatic children treated with steroids without sacrificing asthma control.

Methods. In this case series, treatment of six hypocortisolaemic patients was modified by introducing steroid-sparing asthma medications. Serum cortisol and repeat overnight metyrapone tests (ONMTPTs) were done until HPAS was reversed in all patients. A retrospective folder review was performed and the following data were extracted: body mass index standard deviation score (BMI SDS), adherence, daily steroid type and dose, treatment modification, serum cortisol, final ONMTPT result and time taken to achieve normalisation.

Results. The median serum cortisol level recovered to $311 \mathrm{nmol} / \mathrm{L}$ after 0.9 years (median). The ONMTPT normalised within 3.3 years (median). Steroid load decreased from 9.2 to 5.0 hydrocortisone equivalent $\mathrm{mg} / \mathrm{m}^{2} / \mathrm{d}$ (medians), while asthma score improved from 1.42 to 0.85 (medians). Poor adherence was noted in two children before and four after treatment modification. BMI SDS decreased from -0.08 to -0.16 (medians).

Conclusions. Hypocortisolaemia and HPAS could be reversed in asthmatic children treated with physiological doses of steroids by reducing steroid load by $40 \%$ and supplementing therapy with steroid-sparing medication. Poor adherence may have either contributed to or retarded HPA recovery. Simultaneously, asthma control improved. Confirmation by a prospective study would be ideal, but may not be feasible.

S Afr Med J 2018;108(6):502-505. DOI:10.7196/SAMJ.2018.v108i6.13031

In a multicentre study of 143 asthmatic children treated with inhaled corticosteroids (ICS) with or without nasal steroids (NS), two-thirds were found to have biochemical hypothalamic-pituitaryadrenal axis suppression (HPAS). With the exception of eight who were frankly hypocortisolaemic (as defined by an early-morning serum cortisol level $<83 \mathrm{nmol} / \mathrm{L}$ ), all were assessed by the overnight metyrapone test (ONMTPT). ${ }^{[1]}$ Predictors of HPAS were NS use (odds ratio 3.7 (logistic regression)), body mass index (BMI), and adherence to ICS and NS $(p=0.48, p<0.001$ and $p=0.002$, respectively (linear regression)). At physiological doses (equating a cortisol production rate of $3.0-10.6 \mathrm{mg} / \mathrm{m}^{2} / \mathrm{d}$ (mean (2 standard deviations $)^{[2]}$ ) or a budesonide equivalent of $\left.296-848 \mu \mathrm{g} / \mathrm{m}^{2} / \mathrm{d}\right)$, the post-metyrapone adrenocorticotropic hormone (ACTH) level was inversely correlated to the daily ICS + NS dose, adjusted for body surface area $(r=-0.29, p<0.002)$. At supraphysiological doses this correlation was lost.

All hypocortisolaemic patients were to be started on hydrocortisone (HC) replacement of $8 \mathrm{mg} / \mathrm{m}^{2} / \mathrm{d}$, increasing doses during stress. Subsequently steroid-sparing medications, i.e. longacting beta-agonists, leukotriene receptor antagonists or longacting theophyllines, were to be introduced, followed by a reduction of ICS doses (omalizumab was not available to the South African public sector). To prevent suppression of the early-morning ACTH surge through nocturnal dosing, single morning doses of ICS were recommended. Nasal beclomethasone dipropionate was to be replaced by a single morning dose of a newer-generation NS, e.g. fluticasone propionate. After about a year, asthma control and adherence permitting, HC doses were to be discontinued and earlymorning serum cortisol repeated. If normal, the patients were to undergo a repeat ONMTPT.

\section{Objectives}

To establish whether hypocortisolaemia and HPAS in asthmatic children on steroids could be completely reversed by therapy modification without sacrificing asthma control.

\section{Methods}

After $\sim 1$ year of HC replacement and treatment modification, HC administration to the eight hypocortisolaemic patients seen at the asthma clinics of Red Cross War Memorial Children's Hospital or Tygerberg Hospital was discontinued and their early-morning serum cortisol repeated, provided their asthma was well controlled and the ICS and NS dose stable for 3 - 6 months. If not, doses were increased and testing deferred. After serum cortisol normalised, modification of asthma therapy continued and, asthma control permitting, the ONMTPT was repeated. An adequate response was defined by a rise of ACTH from baseline to $>106 \mathrm{pg} / \mathrm{mL}(23.3 \mathrm{pmol} / \mathrm{L})^{[3]}$ and an 11-deoxycortisol (11DOC) rise to $>208 \mathrm{nmol} / \mathrm{L} .{ }^{[4]}$ If the response was inadequate, ONMTPTs were repeated at $\sim 6$-monthly intervals until adequate. A retrospective folder review was then performed. The following data were extracted: BMI standard deviation score (SDS), asthma score, $\mathrm{FEV}_{1}$ (forced expiratory volume in 1 second), adherence to therapy, daily steroid type and dose, treatment modification, serum cortisol, final ONMTPT result and time taken until normalisation. The asthma score was determined using the Asthma Control Questionnaire, which defines optimal control as a score $<0.75 .^{[5]}$ During the previous study, adherence was formally assessed by enquiring how many doses had been omitted in the preceding week. ${ }^{[1]}$ In the clinical setting, poor adherence was defined as an admission by a child of having omitted any number of doses during the preceding week. The patients' inhalation technique was evaluated and, if 
necessary, corrected during every clinic visit. The total daily steroid load was expressed as $\mathrm{HC}$ equivalent. ${ }^{[1]}$

\section{Ethical approval}

This case series is part of a larger study approved by the Health Research Ethics Committee of Stellenbosch University (ref. no. N06/09/184) and the Research Ethics Committee of the University of Cape Town (ref. no. 276/2003). The original consent included possible treatment modification. Consent for repeat metyrapone testing was reobtained in each case. The Health Research Ethics Committee required no additional consent (all retested patients were from Tygerberg Hospital).

\section{Results}

Eight patients with hypocortisolaemia were identified during the previous study. Of these, one was lost to follow-up and one was excluded because a repeat ONMTPT was not done. The remaining three boys and three girls (all of mixed ancestry) were followed up by the author at the same centre (Tygerberg Children's Hospital). The ages ranged from 5.2 to 12.9 years (mean 8.8 , median 8.3 ). Before hypocortisolaemia was diagnosed, the children had been on ICS for 0.3 - 9.1 years (mean 3.3, median 2.1) and the number of prednisone courses received ranged from 0 to 7 (mean 4, median 5). BMI, asthma control, therapy and modification over time are listed in Table 1. Height and height velocity SDS on follow-up are not reported, because these were previously shown not to correlate with the postmetyrapone ACTH. ${ }^{[1,6]}$ Daily steroid load and HPA function over time are shown in Table 2. HC treatment duration was 3 - 30 months.

By the last follow-up visit, the median daily steroid load had decreased by $\sim 40 \%$ but increased in one child who was not adhering to therapy (Table 2). At the same visit, the asthma score of the group had improved by about the same percentage, but the $\mathrm{FEV}_{1}$ remained similar (Table 1). ICS doses were decreased in all the children except one with poor adherence. NS doses increased overall, partly because good alternatives were not available and partly because of poor compliance (Table 1). In patient 3 , treated with montelukast, NS could be discontinued, while in patient 4 the dose could be reduced on changing to mometasone furoate.

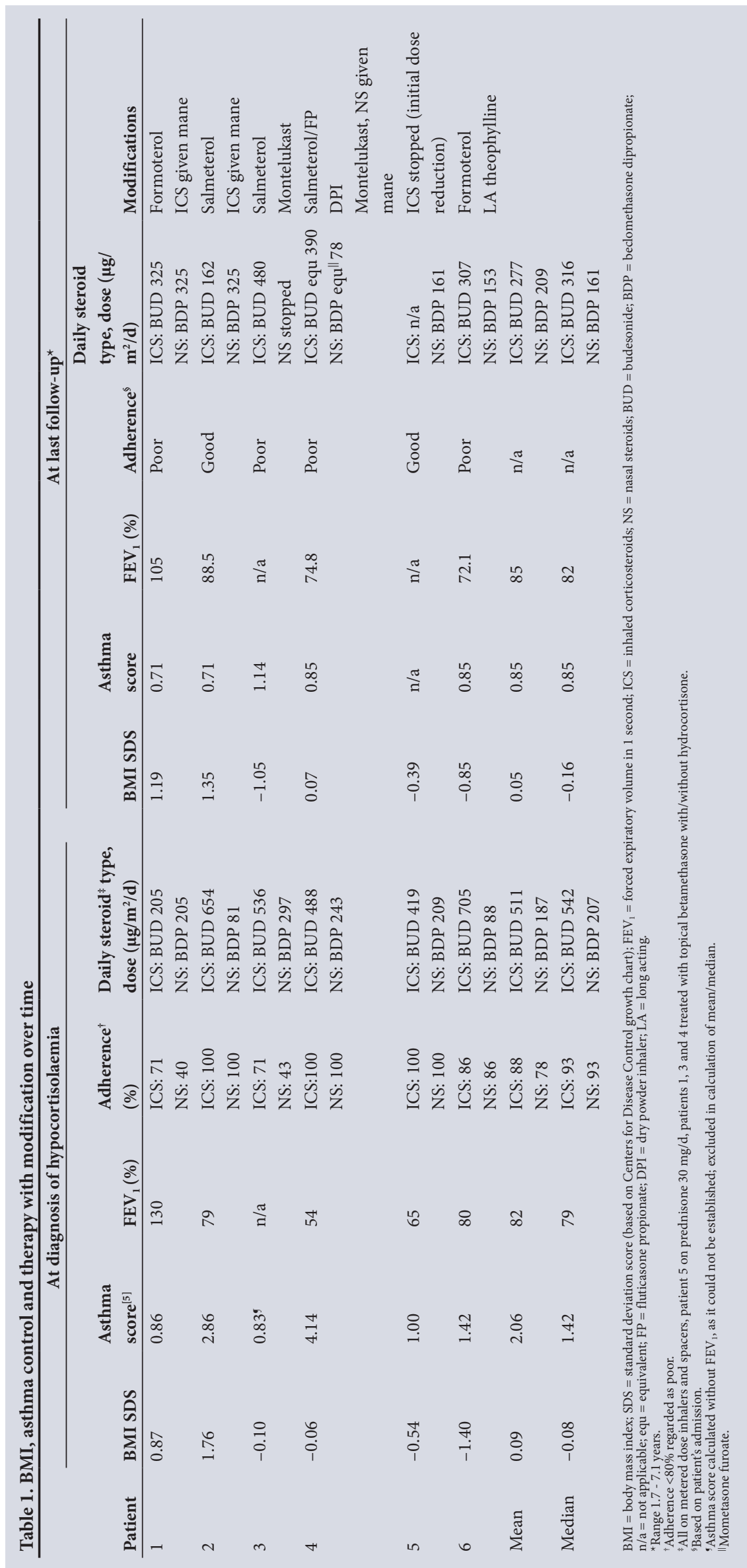


Patient 4's asthma control deteriorated on stopping HC, probably owing to poor adherence to ICS + NS therapy, necessitating a 3-day course of prednisone and an increase in the ICS dose. Patient 3 required a 5-day course of prednisone during an exacerbation, because the mother had discontinued the prescribed HC. Adherence to ICS + NS in this case was also poor, necessitating a second (3-day) course of prednisone, prolonged HC cover and increased ICS doses. Consequently, recovery of the axis took the longest in patients 3 and 4 (Table 2).

Patients 1, 3 and 4 were treated with topical betamethasone with or without HC for atopic dermatitis. Treatment modification in patients 1 and 3 consisted of methylprednisolone aceponate initially, followed by $10 \%$ diluted betamethasone ointment alternating with liquor picis carbonis. After modification, patient 4 only required emollients.

Serum cortisol normalised in all patients (Table 2). There was an adequate ACTH rise on the ONMTPT in all except patient 4, where serum was insufficient. Normalisation of ACTH response can be inferred here, since the hypothalamus and pituitary are known to recover before the adrenal glands. ${ }^{[7]}$ Judging by the adequate $11 \mathrm{DOC}$ rise, these had recovered. The post-metyrapone 11DOC normalised in all except patient 3. A repeat test confirming total recovery was not considered necessary, because the supranormal ACTH response implied imminent recovery and the 11DOC result was approaching normality.

\section{Discussion}

Only one of the children presenting with hypocortisolaemia was subjected to a supraphysiological daily steroid load. All others were treated with a total steroid dose equal to the daily physiological production rate, albeit in the upper range. When disregarding the NS contribution, the low ICS dose would generally be deemed safe. ${ }^{[8]}$ Hypocortisolaemia has, however, been described in children being treated with doses as low as $200 \mu \mathrm{g}$ beclomethasone dipropionate/ day ( $2.5 \mathrm{mg} \mathrm{HC}$ equivalent) or $400 \mu \mathrm{g}$ budesonide/day ( $5 \mathrm{mg} \mathrm{HC}$ equivalent). ${ }^{[9,10]}$ The suppressed cortisol production is balanced by the supply of exogenous steroids. Hypocortisolaemia is one step away from adrenal crisis. When cortisol demand outstrips exogenous supply, as during an asthma exacerbation, an infection, surgery, injury or a burn, a crisis can be precipitated. As these stressors are usually unpredictable, $\mathrm{HC}$ replacement was prescribed as a precaution against a possible crisis. It also allowed for safe modification of asthma therapy. Furthermore, HC replacement was a protocol requirement, being essential therapy for hypocortisolaemia.

After modification, the median steroid load of the group decreased by $\sim 40 \%$, leading to HPAS reversal in all cases. Instead of deteriorating, asthma control actually improved. In addition, the number of rescue prednisone courses required decreased considerably. The steroid/ non-steroid combination therefore appears to be superior in both safety and efficacy compared with the steroid-only therapy given before the intervention. Polypharmacy did not seem to have affected outcomes adversely. This case series formally documents the reversal of HPAS caused by low steroid doses, and the time taken for reversal associated with improved asthma control. To the author's knowledge this has not been described previously. The limitations of a retrospective report are counterbalanced by a single clinician managing all aspects of all cases. Ideally the findings of this case series need to be confirmed in a well-designed prospective study, but this may be difficult to obtain ethical approval for or to implement.

Could factors other than treatment modification have contributed to the recovery of HPAS in these cases? NS use, BMI and adherence to ICS and NS have been identified previously. ${ }^{[1]}$ Firstly, NS could only be

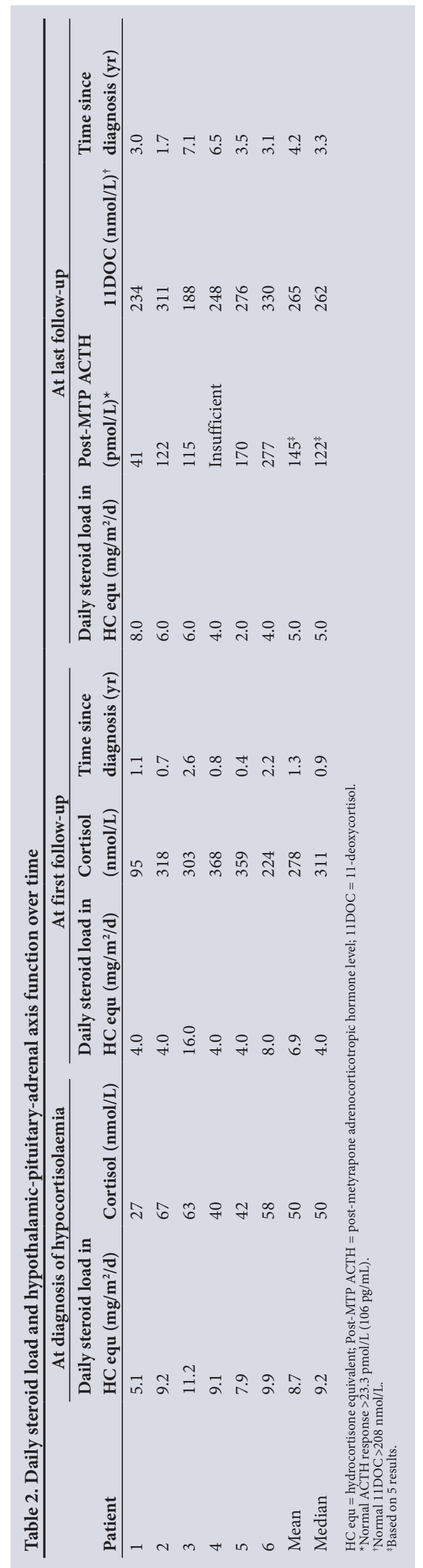


discontinued or reduced in two cases. A change in NS use is unlikely to have affected outcome, however, because median NS dosage actually increased. Secondly, the group's BMI did not increase over time, implying that a BMI change is not likely to have contributed to recovery. Thirdly, although the assessment of adherence was limited by the different methods used before and after intervention, poor adherence could have contributed to the reversal in the two additional children known to have been poorly compliant after treatment modification. The reason for the poor adherence is not known, but is likely to be related to inadequate supervision due to the children's advancing age. On the other hand, it also retarded recovery, as exacerbations necessitated prednisone courses and higher ICS doses, albeit intermittently. Furthermore, as airway diameter increases with age, airway narrowing is less severe, resulting in improved asthma control with subsequent lower ICS dose and recovery of the axis. However, while asthma was well controlled, dosages were reduced to lower steroid exposure and not because of improved symptoms.

\section{Conclusions}

In this case series, hypocortisolaemia developed in asthmatic children while all but one were being treated with steroid doses in the physiological range. On reducing the daily steroid load by $\sim 40 \%$, hypocortisolaemia and HPAS were effectively reversed in all. Poor adherence may have either contributed to or retarded HPA recovery. Simultaneously, asthma control improved. A prospective study would be ideal to confirm these findings, but may not be feasible.

\section{Acknowledgements. None.}

Author contributions. The author conceptualised and designed the case series and the study it is based on. He analysed and interpreted the data and wrote the article.

Funding. None.

Conflicts of interest. None.

1. Zöllner EW, Lombard CJ, Hough FS, et al. Hypothalamic-pituitary-adrenal axis suppression in asthmatic school children. Pediatrics 2012;130(6):e1512-e1519. https://doi.org/10.1542/peds.2012-1147 2. Linder BL, Esteban NV, Yergey AL, et al. Cortisol production rate in childhood and adolescence. J Pediatr 1990;117(6):892-896. https://doi.org/10.1016/S0022-3476(05)80128-3

3. Staub JJ, Noelpp B, Girard J, Baumann JB, Graf S, Ratcliffe JG. The short metyrapone test: Comparison of the plasma ACTH response to metyrapone and insulin-induced hypoglycaemia. Clin Endocrinol 1979;10(6):595-601. https://doi.org/10.1111/j.1365-2265.1979.tb02119x

4. Fiad TM, Kirby JM, Cunningham SK, McKenna TJ. The overnight single-dose metyrapone test is a simple and reliable index of the hypothalamic-pituitary-adrenal axis. Clin Endocrinol 1994;40(5):603609. https://doi.org/10.1111/j.1365-2265.1994.tb03011.x

5. Juniper EF, Bousquet J, Abetz L, Bateman ED; The GOAL Committee. Identifying 'well-controlled' and 'not well-controlled' asthma using the Asthma Control Questionnaire. Respir Med 2006;100(4):616-621. https://doi.org/10.1016/.r.rmed.2005.08.012

6. Zöllner EW, Lombard C, Galal U, Hough S, Irusen E, Weinberg E. Hypothalamic-pituitary-adrenal axis suppression in asthmatic children on inhaled and nasal corticosteroids - more common than expected? J Pediatr Endocrinol Metab 2011;24(7-8):529-534. https://doi.org/10.1515/jpem.2011.198

7. Graber AL, Ney RL, Nicholson WE, et al. Natural history of pituitary-adrenal recovery following longterm suppression with corticosteroids. J Clin Endocrinol 1965;25(Jan):11-16.

8. Issa-El-Khoury K, Kim H, Chan ES, et al. CSAI position statement: Systemic effect of inhaled corticosteroids on adrenal suppression in the management of pediatric asthma. Allergy Asthma Clin Immunol 2015;11(1):9. https://doi.org/10.1186/s13223-015-0075-z

9. Shenoy SD, Swift PGF, Cody D. Growth impairment and adrenal suppression on low-dose inhaled beclomethasone. J Paediatr Child Health 2006;42(3):143-144. https://doi.org/10.1111/j.14401754.2006.00814.x

10. Patel L, Wales JK, Kibirige MS, Massarano AA, Couriel JM, Clayton PE. Symptomatic adrenal insufficiency during inhaled corticosteroid treatment. Arch Dis Child 2001;85(4):330-334. https://doi. org/10.1136/adc.85.4.330

Accepted 18 January 2018 